\begin{tabular}{|c|l|}
\hline Title & The composition operators on weighted bloch space \\
\hline Author(s) & Yoneda, R. \\
\hline Citation & Hokkaido University Preprint Series in Mathematics, 517, 1-8 \\
\hline Issue Date & 2001-1-1 \\
\hline DOI & 10.14943/83663 \\
\hline Doc URL & http://hdl.handle.net/2115/69267 \\
\hline Type & bulletin (article) \\
\hline File Information & pre517.pdf \\
\hline
\end{tabular}

Instructions for use 
The Composition Operators On Weighted Bloch Space

Rikio Yoneda

Series \#517. January 2001 


\section{HOKKAIDO UNIVERSITY PREPRINT SERIES IN MATHEMATICS}

\#492 I. Tsuda and M. Hatakeyama, Making sense of internal logic: Theory and a case study, 10 pages. 2000.

\#493 I. Tsuda, Towards an interpretation of dynamic neural activity in terms of chaotic dynamical systems, 73 pages. 2000 .

\#494 T. Mikami, Optimal control for absolutely continuous stochastic processes and the mass transportation problem, 17 pages. 2000 .

\#495 M. Arisawa and Y. Giga, Anisotropic curvature flow in a very thin domain, 21 pages. 2000.

\#496 T. Nakazi, Backward shift invariant subspaces in the bidisc, 9 pages. 2000.

\#497 Y. Giga, K. Inui, J. Kato and S. Matsui, Remarks on the uniqueness of bounded solutions of the NavierStokes equations, 4 pages. 2000.

\#498 Y. Giga, M. Paolini and P. Rybka, On the motion by singular interfacial energy, 21 pages. 2000.

\#499 J. Escher and Y. Giga, On a limiting motion and self-interactions of curves moved by the intermediate surface diffusion flow, 12 pages. 2000.

\#500 I. Tsuda and S. Kuroda, Cantor coding in the hippocampus, 20 pages. 2000.

\#501 M. Tsujii, Fat solenoidal attractors, 20 pages. 2000.

\#502 A. Arai, Ground state of the massless Nelson model without infrared cutoff in a non-Fock representation, 19 pages. 2000.

\#503 Y. Giga, S. Matsui and O. Sawada, Global existence of two-dimensional Navier-Stokes flow with nonde caying initial velocity, 19 pages. 2000 .

\#504 A. Inoue and Y. Kasahara, Partial autocorrelation functions of the fractional ARIMA processes with negative degree of differencing, 14 pages. 2000.

\#505 T. Nakazi, Interpolation problem for $\ell^{1}$ and a uniform algebra, 12 pages. 2000.

\#506 R. Kobayashi and Y. Giga, On anisotropy and curvature effects for growing crystals, 38 pages. 2000.

\#507 A. Arai, Instability in the spectral and the Fredholm properties of an infinite dimensional Dirac operator on the abstract Boson-Fermion Fock space, 6 pages. 2000.

\#508 A. Arai, Supersymmetric methods for constructing soliton-type solutions to multi-component nonlinear Schrödinger and Klein-Gordon equations, 22 pages. 2000.

\#509 Y. Tonegawa, Phase field model with a variable chemical potential, 30 pages. 2000.

\#510 Y. Giga, Shocks and very strong vertical diffusion, 11 pages. 2000.

\#511 S. Izumiya \& N. Takeuchi Special curves and raled surfaces, 18 pages. 2001.

\#512 S. Izumiya Generating families of developable surfaces in $R^{3}, 18$ pages. 2001.

\#513 S. Izumiya, K. Maruyama Transversal topology and singularities of Haefliger foliations, 8 pages. 2001.

\#514 S. Izumiya, D-H. Pei \& T. Sano Singularities of hyperbolic Gauss maps, 27 pages. 2001.

\#515 S. Izumiya, N. Takeuchi Generic special curves, 12 pages. 2001.

\#516 S. Izumiya. D-H. Pei \& T. Sano Horospherical surfaces of curves in hyperbolic space, 9 pages. 2001. 


\title{
The Composition Operators On Weighted Bloch Space
}

\author{
Rikio Yoneda
}

\begin{abstract}
We will characterize the boundedness and compactness of the composition operators on weighted Bloch space $B_{\log }=\left\{f \in H(D): \sup _{z \in D}\left(1-|z|^{2}\right)\left(\log \frac{2}{1-|z|^{2}}\right)\left|f^{\prime}(z)\right|<+\infty\right\}$, where $H(D)$ be the class of all analytic functions on $D$.
\end{abstract}

Key Words and Phrases : composition operator, weighted Bloch space, compactness, boundedness.

\section{$\S 1$. Introduction}

Let $D$ denote the open unit disk in complex plane $C$ and $\partial D$ denote the unit circle in $C$. For $1 \leq p<+\infty$, the Lebesgue space $L^{p}(D, d A)$ is defined to be the Banach space of Lebesgue measurable functions on $D$ with

$$
\|f\|_{L^{p}(d A)}:=\left(\int_{D}|f(z)|^{p} d A(z)\right)^{\frac{1}{p}}<+\infty,
$$

where $d A(z)$ is the normalized area measure on $D$. The Bergman space $L_{a}^{p}(D)$ is defined to be the subspace of $L^{p}(D, d A)$ consisting of analytic functions. The orthogonal projection from $L^{2}(D, d A)$ onto $L_{a}^{2}$ is called the Bergman projection and is denoted by $P$. Then given a function $f \in L^{2}(D, d A)$, an operator $H_{f}: L_{a}^{2} \rightarrow\left(L_{a}^{2}\right)^{\perp}$ is defined by $H_{f} g=(I-P)(f g), g \in L_{a}^{2}$. The operator $H_{f}$ is called the Hankel operator on the Bergman space with symbol $f$.

For $0<p<+\infty$, the Hardy space $H^{p}$ is defined to be the Banach space of analytic functions $f$ on $D$ with

$$
\|f\|_{p}:=\left(\sup _{0<r<1} \frac{1}{2 \pi} \int_{0}^{2 \pi}\left|f\left(r e^{i \theta}\right)\right|^{p} d \theta\right)^{\frac{1}{p}}<+\infty .
$$

The orthogonal projection from $L^{2}(\partial D)$ onto $H^{2}$ is called the Szegö projection and is also denoted by $P$. Then given a function $f \in L^{2}(\partial D)$, an operator $H_{f}: H^{2} \rightarrow\left(H^{2}\right)^{\perp}$ is defined by $H_{f} g=(I-P)(f g), g \in H^{2}$. The operator $H_{f}$ is called the Hankel operator on the Hardy space with symbol $f$.

The Bloch space $B$ of $D$ is defined to be the space of analytic functions $f$ on $D$ such that

$$
\|f\|_{B}:=\sup _{z \in D}\left(1-|z|^{2}\right)\left|f^{\prime}(z)\right|<+\infty .
$$

2000 Mathematics Subject Classification : Primary 47 B33. 
The space of analytic functions on $D$ of bounded mean oscillation, denoted by $B M O A$, consists of functions $f$ in $H^{2}$ for which

$$
\|f\|_{B M O A}:=\sup _{I} \frac{2}{|I|} \int_{S(I)}\left|f^{\prime}(z)\right|^{2} \log \frac{1}{|z|} d A(z)<+\infty,
$$

where $I$ denotes a subarc of $\partial D,|I|$ denotes the arclength measure of $I$, and $S(I)=\left\{r e^{i \theta}\right.$ : $\left.1-r \leq|I|, e^{i \theta} \in I\right\}$ ( see [6] ).

And the following are the classical results for the spaces $B$ and $B M O A$ ( see [9] ): For $f \in L_{a}^{2}$, Hankel operator $H_{\bar{f}}: L_{a}^{2} \rightarrow\left(L_{a}^{2}\right)^{\perp}$ is bounded if and only if $f \in B$. And for $f \in H^{2}$, Hankel operator $H_{\bar{f}}: H^{2} \rightarrow\left(H^{2}\right)^{\perp}$ is bounded if and only if $f \in B M O A$.

It is well-known that $B M O A \subset B$ ( see [6] or [9] ). It is called that a holomorphic self map $\varphi$ of $D$ has Bloch-to-BMOA pullback property if $f \circ \varphi \in B M O A$ for all $f \in B$. In [3], B.R.Choe, W.Ramey,and D.Ullrich studied Bloch-to- $B M O A$ pullbacks.

On the other hand, in [1], K.R.M. Attle proved that for $f \in L_{a}^{2}(D)$, the Hankel operator $L_{a}^{1} \rightarrow L^{1}$ is bounded if and only if

$$
\|f\|_{B_{\log }}:=\sup _{z \in D}\left(1-|z|^{2}\right)\left(\log \frac{2}{1-|z|^{2}}\right)\left|f^{\prime}(z)\right|<+\infty
$$

and in [4], Cima and Stegenga proved that the Hankel operator $H_{f}: H^{1} \rightarrow H^{1}$, with an analytic symbol $f$ (see their paper for the definition of this Hankel operator) is bounded if and only if

$$
\|f\|_{B M O A_{\log }}:=\sup _{I} \frac{\left(\log \frac{2}{|I|}\right)^{2}}{|I|} \int_{S(I)}\left|f^{\prime}(z)\right|^{2} \log \frac{1}{|z|} d A(z)<+\infty .
$$

Accordingly, we define the weighted Bloch space $B_{\log }$ of $D$ to be the space of analytic functions $f$ on $D$ such that $\|f\|_{B_{\log }}<+\infty$. And we define the space of analytic functions on $D$ of weighted bounded mean oscillation, denoted by $B M O A_{\log }$, to be the space of functions $f$ in $H^{2}$ for which $\|f\|_{B M O A_{\log }}<+\infty$.

By a direct calculation, we see that $B M O A_{\log } \subset B_{\log }$ (see [6] or [9]). We will also say that a holomorphic self map $\varphi$ of $D$ has $B_{\log }$-to- $B M O A_{\log }$ pullback property if $f \circ \varphi \in B M O A_{\log }$ for all $f \in B_{\log }$.

Let $\varphi$ denote a holomorphic function taking $D$ into $D$ and $C_{\varphi}$ denote the composition operator with $\varphi$. Then it is trivial that $C_{\varphi}$ is bounded on $B$ ( see [7] ). In [7], K.Madigan and A.Matheson showed the following result about the compactness of $C_{\varphi}$ on $B$ :

Theorem $\mathrm{A}$. Let $\varphi$ be a holomorphic function taking $D$ into $D$. Then $C_{\varphi}$ is compact on $B$ if and only if for every $\epsilon>0$, there exists $0<r<1$ such that

$$
\sup _{|\varphi(z)|>r}\left(\frac{\left(1-|z|^{2}\right)}{\left(1-|\varphi(z)|^{2}\right)}\left|\varphi^{\prime}(z)\right|\right)<\epsilon .
$$

In this paper, we study the boundedness and compactness of the composition operator on $B_{\log }$. Moreover by using $B_{\log }$-to- $B M O A_{\log }$ pullback property, we also study the composition operator on $B M O A_{\log }$. Throughout this paper, $C_{i}, K_{i}$ for $i=0,1,2, C, K$ will denote positive constant whose value is not necessary the same at each occurrence. 
$\S 2$. The composition operator on the weighted Bloch space $B_{\log }$

In this section, we study the boundedness and compactness of the composition operator on $B_{\log }$.

Theorem 1. Let $\varphi$ be a holomorphic function taking $D$ into $D$. Then $C_{\varphi}$ is bounded on $B_{\log }$ if and only if $\sup _{z \in D}\left(\frac{\left(1-|z|^{2}\right) \log \frac{2}{1-|z|^{2}}}{\left(1-|\varphi(z)|^{2}\right) \log \frac{2}{1-|\varphi(z)|^{2}}}\left|\varphi^{\prime}(z)\right|\right)<+\infty$.

proof. Suppose that $\sup _{z \in D}\left(\frac{\left(1-|z|^{2}\right) \log \frac{2}{1-|z|^{2}}}{\left(1-|\varphi(z)|^{2}\right) \log \frac{2}{1-|\varphi(z)|^{2}}}\left|\varphi^{\prime}(z)\right|\right)<+\infty . \quad$ Let $f$ be in the space $B_{\text {log }}$. Then we see that

$$
\begin{aligned}
& \sup _{z \in D}\left|(f \circ \varphi)^{\prime}(z)\right|\left(1-|z|^{2}\right) \log \frac{2}{1-|z|^{2}} \\
& =\sup _{z \in D} \mid\left(f^{\prime}(\varphi(z))|| \varphi^{\prime}(z) \mid\left(1-|z|^{2}\right) \log \frac{2}{1-|z|^{2}}\right. \\
& \leq \sup _{z \in D} \mid\left(f^{\prime}(\varphi(z)) \mid\left(1-|\varphi(z)|^{2}\right)\left(\log \frac{2}{1-|\varphi(z)|^{2}}\right) \sup _{z \in D}\left(\frac{\left(1-|z|^{2}\right) \log \frac{2}{1-|z|^{2}}}{\left(1-|\varphi(z)|^{2}\right) \log \frac{2}{1-|\varphi(z)|^{2}}}\left|\varphi^{\prime}(z)\right|\right)\right. \\
& \leq C\|f\|_{B_{\log }} .
\end{aligned}
$$

To prove the converse, suppose that $C_{\varphi}$ is bounded on $B_{\text {log }}$. Then $\|f \circ \varphi\|_{B_{\log }} \leq C\|f\| B_{\text {log }}$ for all $f \in B_{\log }$. For $w \neq 0$, let $f_{w}$ be the anti-derivative of

$$
\left(1-\frac{\bar{w}^{2}}{|w|^{2}} z^{2}\right)^{-1}\left(\log \frac{2}{1-\frac{\bar{w}^{2}}{|w|^{2}} z^{2}}\right)^{-1}
$$

with $f_{w}(0)=0$. Since $\sup _{z_{1} \in D}\left(1-\left|z_{1}\right|^{2}\right)\left(\log \frac{2}{1-\left|z_{1}\right|^{2}}\right)\left|1-z_{1}^{2}\right|^{-1}\left|\log \frac{2}{1-z_{1}^{2}}\right|^{-1}<+\infty$, applying $z_{1}=\frac{\bar{w}}{|w|} z$, we have

$$
\sup _{z \in D}\left(1-|z|^{2}\right)\left(\log \frac{2}{1-|z|^{2}}\right)\left|1-\frac{\bar{w}^{2}}{|w|^{2}} z^{2}\right|^{-1}\left|\log \frac{2}{1-\frac{\bar{w}^{2}}{|w|^{2}} z^{2}}\right|^{-1}<+\infty \text {. }
$$

Hence we have $f_{w} \in B_{\log }$ for $w \neq 0 . \quad$ Since $C_{\varphi}$ is bounded on $B_{\log }$, we have $\left\|f_{w} \circ \varphi\right\|_{B_{\log }}<$ $+\infty$. Thus for $w \neq 0$,

$$
\sup _{z \in D}\left(1-|z|^{2}\right)\left(\log \frac{2}{1-|z|^{2}}\right)\left|\left(f_{w} \circ \varphi\right)^{\prime}(z)\right| \leq K<+\infty .
$$


For any $z \in D$ and $w \neq 0 \in D$, we have $\left(1-|z|^{2}\right)\left(\log \frac{2}{1-|z|^{2}}\right)\left|f_{w}^{\prime}(\varphi(z))\right|\left|\varphi^{\prime}(z)\right| \leq K$. Fix an arbitrary $z \in D$ with $\varphi(z) \neq 0$, applying $w=\varphi(z)$ to the above inequality, we have

$$
\left(1-|z|^{2}\right)\left(\log \frac{2}{1-|z|^{2}}\right)\left|1-\frac{\overline{\varphi(z)}^{2}}{|\varphi(z)|^{2}} \varphi(z)^{2}\right|^{-1}\left|\log \frac{2}{1-\frac{\overline{\varphi(z)}^{2}}{|\varphi(z)|^{2}} \varphi(z)^{2}}\right|^{-1}\left|\varphi^{\prime}(z)\right| \leq K<\infty .
$$

Hence for an arbitrary $z \in D$ with $\varphi(z) \neq 0, \frac{\left(1-|z|^{2}\right) \log \frac{2}{1-|z|^{2}}}{\left(1-|\varphi(z)|^{2}\right) \log \frac{2}{1-|\varphi(z)|^{2}}}\left|\varphi^{\prime}(z)\right| \leq K<+\infty$. For an arbitrary $z \in D$ with $\varphi(z)=0$, since $\varphi \in B_{\log }$, we have

$$
\left(\frac{\left(1-|z|^{2}\right) \log \frac{2}{1-|z|^{2}}}{\left(1-|\varphi(z)|^{2}\right) \log \frac{2}{1-|\varphi(z)|^{2}}}\left|\varphi^{\prime}(z)\right|\right)=\frac{1}{\log 2}\left(1-|z|^{2}\right)\left(\log \frac{2}{1-|z|^{2}}\right)\left|\varphi^{\prime}(z)\right|<+\infty .
$$

This completes the proof of the theorem.

For example, for $z, w \in D, \varphi(z)=\frac{w-z}{1-\bar{w} z}$ satisfies the condition of Theorem 1

Theorem 2. Let $\varphi$ be a holomorphic function taking $D$ into $D$. Then $C_{\varphi}$ is compact on $B_{\log }$ if and only if $\varphi \in B_{\log }$ and for every $\epsilon>0$ there exists $0<r<1$ such that

$$
\sup _{|\varphi(z)|>r}\left(\frac{\left(1-|z|^{2}\right) \log \frac{2}{1-|z|^{2}}}{\left(1-|\varphi(z)|^{2}\right) \log \frac{2}{1-|\varphi(z)|^{2}}}\left|\varphi^{\prime}(z)\right|\right)<\epsilon .
$$

proof. Suppose that $\varphi \in B_{\log }$ and for every $\epsilon>0$ there exists $0<r<1$ such that

$$
\sup _{|\varphi(z)|>r}\left(\frac{\left(1-|z|^{2}\right) \log \frac{2}{1-|z|^{2}}}{\left(1-|\varphi(z)|^{2}\right) \log \frac{2}{1-|\varphi(z)|^{2}}}\left|\varphi^{\prime}(z)\right|\right)<\epsilon .
$$

Suppose that $f_{n}$ is bounded sequence in $B_{\log }$ converging to 0 uniformly on compact subsets of

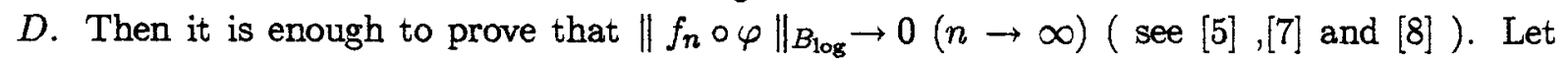
$K:=\sup _{n}\left\|f_{n}\right\|_{B_{\text {log }}}$. For any $\epsilon>0$, there is $0<r<1$ such that

$$
\frac{\left(1-|z|^{2}\right) \log \frac{2}{1-|z|^{2}}}{\left(1-|\varphi(z)|^{2}\right) \log \frac{2}{1-|\varphi(z)|^{2}}}\left|\varphi^{\prime}(z)\right|<\frac{\epsilon}{2 K}
$$

whenever $|\varphi(z)|>r$. So we have

$$
\begin{aligned}
& \left(1-|z|^{2}\right)\left(\log \frac{2}{1-|z|^{2}}\right)\left|\left(f_{n} \circ \varphi\right)^{\prime}(z)\right| \\
= & \frac{\left(1-|z|^{2}\right) \log \frac{2}{1-|z|^{2}}}{\left(1-|\varphi(z)|^{2}\right) \log \frac{2}{1-|\varphi(z)|^{2}}}\left|\varphi^{\prime}(z)\right|\left(1-|\varphi(z)|^{2}\right)\left(\log \frac{2}{1-|\varphi(z)|^{2}}\right)\left|f_{n}^{\prime}(\varphi(z))\right| \leq \frac{\epsilon}{2 K} K=\frac{\epsilon}{2} .
\end{aligned}
$$


Hence $\left(1-|z|^{2}\right)\left(\log \frac{2}{1-|z|^{2}}\right)\left|\left(f_{n} \circ \varphi\right)^{\prime}(z)\right|<\frac{\epsilon}{2}$ whenever $|\varphi(z)|>r$.

On the other hand, $f_{n} \circ \varphi(0) \rightarrow 0$ and $\left(1-|w|^{2}\right)\left(\log \frac{2}{1-|w|^{2}}\right)\left|f_{n}^{\prime}(w)\right| \rightarrow 0$ for $|w| \leq r($ $n \rightarrow+\infty)$. By the assumption $\varphi \in B_{\log }$, we have

$$
\sup _{|\varphi(z)| \leq r}\left(\frac{\left(1-|z|^{2}\right) \log \frac{2}{1-|z|^{2}}}{\left(1-|\varphi(z)|^{2}\right) \log \frac{2}{1-|\varphi(z)|^{2}}}\left|\varphi^{\prime}(z)\right|\right) \leq C\|\varphi\|_{B_{\log }}<+\infty .
$$

So put $K_{1}:=\sup _{|\varphi(z)| \leq r}\left(\frac{\left(1-|z|^{2}\right) \log \frac{2}{1-|z|^{2}}}{\left(1-|\varphi(z)|^{2}\right) \log \frac{2}{1-|\varphi(z)|^{2}}}\left|\varphi^{\prime}(z)\right|\right)$. Then for $\frac{\epsilon}{K_{1}}>0$ and for large enough $n$,

$$
\left(1-|w|^{2}\right)\left(\log \frac{2}{1-|w|^{2}}\right)\left|f_{n}^{\prime}(w)\right| \leq \frac{\epsilon}{2 K_{1}} \text { whenever }|w| \leq r \text { and }\left|f_{n} \circ \varphi(0)\right|<\frac{\epsilon}{2} \text {. }
$$

Hence for any $\epsilon>0$ and for large enough $n$,

$$
\begin{aligned}
& \left(1-|z|^{2}\right)\left(\log \frac{2}{1-|z|^{2}}\right)\left|\left(f_{n} \circ \varphi\right)^{\prime}(z)\right| \\
= & \left(1-|z|^{2}\right)\left(\log \frac{2}{1-|z|^{2}}\right)\left|f_{n}^{\prime}(\varphi(z))\right|\left|\varphi^{\prime}(z)\right| \\
= & \left(1-|\varphi(z)|^{2}\right)\left(\log \frac{2}{1-|\varphi(z)|^{2}}\right)\left|f_{n}^{\prime}(\varphi(z))\right| \frac{\left(1-|z|^{2}\right) \log \frac{2}{1-|z|^{2}}}{\left(1-|\varphi(z)|^{2}\right) \log \frac{2}{1-|\varphi(z)|^{2}}}\left|\varphi^{\prime}(z)\right| \leq \frac{\epsilon}{2 K_{1}} K_{1}=\frac{\epsilon}{2} .
\end{aligned}
$$

Hence we have for any $\epsilon>0$ and for large enough $n$,

$$
\left|f_{n} \circ \varphi(0)\right|+\left(1-|z|^{2}\right)\left(\log \frac{2}{1-|z|^{2}}\right)\left|\left(f_{n} \circ \varphi\right)^{\prime}(z)\right|<\frac{\epsilon}{2}+\frac{\epsilon}{2}=\epsilon .
$$

To prove the converse, suppose that there exists a sequence $\left\{z_{n}\right\}_{n} \subset D$ and for some $\epsilon_{0}>0$ such that $\left|z_{n}\right| \rightarrow 1^{-}$and $\left(\frac{\left(1-|z|^{2}\right) \log \frac{2}{1-|z|^{2}}}{\left(1-|\varphi(z)|^{2}\right) \log \frac{2}{1-|\varphi(z)|^{2}}}\left|\varphi^{\prime}(z)\right|\right)>\epsilon_{0}$. Then we show that $C_{\varphi}$ is not compact. By taking a subsequence, it may be assumed that $w_{n}=\varphi\left(z_{n}\right) \rightarrow w_{0}:=\varphi\left(z_{0}\right) \in \partial D$. Let $f_{n}(z)=\log \left(\log \frac{2}{1-\overline{w_{n} z}}\right)$ and $f_{0, n}(z)=\log \left(\log \frac{2}{1-\overline{w_{0}} e^{i\left(\theta_{0}-\theta_{n}\right)} z}\right)$ where $\theta_{n}=\arg \varphi\left(z_{n}\right)$ and $\theta_{0}=\arg \varphi\left(z_{0}\right)$. Since $\theta_{n} \rightarrow \theta_{0}(n \rightarrow \infty)$, we see that $\left\{g_{n}\right\}_{n}=\left\{f_{n}-f_{0, n}\right\}_{n}$ converges to 0 uniformly on compact subsets of $D$. Since $\frac{\overline{w_{0}} e^{i\left(\theta_{0}-\theta_{n}\right)}}{\overline{w_{n}}} \frac{\left(1-\left|w_{n}\right|^{2}\right) \log \frac{2}{1-\left|w_{n}\right|^{2}}}{\left(1-\left|w_{n}\right|\right) \log \frac{2}{1-\left|w_{n}\right|}} \rightarrow 2(n \rightarrow+\infty)$ and $\left|\varphi\left(z_{n}\right)\right| \rightarrow 1(n \rightarrow+\infty)$, we have for all $n$

$$
\begin{aligned}
& \left\|C_{\varphi} g_{n}\right\|_{B_{\log }}=\left\|C_{\varphi} f_{n}-C_{\varphi} f_{0, n}\right\|_{B_{\log }} \\
& \geq\left(1-\left|z_{n}\right|^{2}\right)\left(\log \frac{2}{1-\left|z_{n}\right|^{2}}\right)\left|\left(C_{\varphi} f_{n}\right)^{\prime}\left(z_{n}\right)-\left(C_{\varphi} f_{0, n}\right)^{\prime}\left(z_{n}\right)\right| \\
& =\left(1-\left|z_{n}\right|^{2}\right)\left(\log \frac{2}{1-\left|z_{n}\right|^{2}}\right)\left|f_{n}^{\prime}\left(\varphi\left(z_{n}\right)\right)-f_{0, n}^{\prime}\left(\varphi\left(z_{n}\right)\right)\right|\left|\varphi^{\prime}\left(z_{n}\right)\right| \\
& =\left(1-\left|z_{n}\right|^{2}\right)\left(\log \frac{2}{1-\left|z_{n}\right|^{2}}\right)\left|\varphi^{\prime}\left(z_{n}\right)\right|\left|\frac{\overline{w_{n}}}{\left(1-\left|w_{n}\right|^{2}\right) \log \frac{2}{1-\left|w_{n}\right|^{2}}}-\frac{\overline{w_{0}} e^{i\left(\theta_{0}-\theta_{n}\right)}}{\left(1-\left|w_{n}\right|\right) \log \frac{2}{1-\left|w_{n}\right|}}\right|
\end{aligned}
$$




$$
\begin{aligned}
& =\left(\frac{\left(1-\left|z_{n}\right|^{2}\right) \log \frac{2}{1-\left|z_{n}\right|^{2}}}{\left(1-\left|\varphi\left(z_{n}\right)\right|^{2}\right) \log \frac{2}{1-\left|\varphi\left(z_{n}\right)\right|^{2}}}\left|\varphi^{\prime}\left(z_{n}\right)\right|\right)\left|\varphi\left(z_{n}\right)\right|\left|1-\frac{\overline{w_{0}} e^{i\left(\theta_{0}-\theta_{n}\right)}}{\overline{w_{n}}} \frac{\left(1-\left|w_{n}\right|^{2}\right) \log \frac{2}{1-\left|w_{n}\right|^{2}}}{\left(1-\left|w_{n}\right|\right) \log \frac{2}{1-\left|w_{n}\right|}}\right| \\
& \geq \epsilon_{0}>0 .
\end{aligned}
$$

Hence we see that $C_{\varphi}$ is not compact. This completes the proof of the theorem.

\section{$\S 3$. The composition operator on weighted $B M O A_{\log }$}

In this section, we study the composition operator on weighted $B M O A_{\mathrm{log}}$. But, by a direct calculation, it is not easy that we do see when $C_{\varphi} f \in B M O A_{\log }$ for $f \in B M O A_{\log }$. So by the following proposition, we can get the condition of a holomorphic function $\varphi$ that $C_{\varphi} f \in B M O A_{\log }$ for $f \in B_{\log }$ holds. In particular, we can get the condition of a holomorphic function $\varphi$ that $C_{\varphi} f \in B M O A_{\log }$ for $f \in B M O A_{\log }$ holds because of $B M O A_{\log } \subset B_{\log }$.

Proposition 3. If $\sup _{I} \frac{\left(\log \frac{2}{|I|}\right)^{2}}{|I|} \int_{S(I)} \frac{\left(1-|z|^{2}\right)\left|\varphi^{\prime}(z)\right|^{2}}{\left(1-|\varphi(z)|^{2}\right)^{2}\left(\log \frac{2}{1-|\varphi(z)|^{2}}\right)^{2}} d A(z)<+\infty$, then $\varphi(z)$ has $B_{\log }$-to- $B M O A_{\log }$ pullback property. Conversely, if there exists a sequence $\left\{w_{n}\right\}_{n=1}^{\infty} \subset$ $\partial D$ such that $\varphi(D) \subset \cup_{n=1}^{\infty}\left\{z \in D:\left|1-\overline{w_{n}} z\right|<\lambda\left(1-|z|^{2}\right)\right\}$ where $\lambda>0$, if $\varphi(z)$ has $B_{\text {log }}$-to$B M O A_{\log }$ pullback property, then

$$
\sup _{I} \frac{\left(\log \frac{2}{|I|}\right)^{2}}{|I|} \int_{S(I)} \frac{\left(1-|z|^{2}\right)\left|\varphi^{\prime}(z)\right|^{2}}{\left(1-|\varphi(z)|^{2}\right)^{2}\left(\log \frac{2}{1-|\varphi(z)|^{2}}\right)^{2}} d A(z)<+\infty
$$

proof. Let $f \in B_{\log }$. Then we have

$$
\begin{aligned}
& \sup _{I} \frac{\left(\log \frac{2}{|I|}\right)^{2}}{|I|} \int_{S(I)}\left|(f \circ \varphi)^{\prime}\right|^{2}\left(1-|z|^{2}\right) d A(z) \\
& \quad \leq \sup _{I} \frac{\left(\log \frac{2}{|I|}\right)^{2}}{|I|} \int_{S(I)}\left|\left(f^{\prime}(\varphi(z))\right)\right|^{2}\left|\varphi^{\prime}(z)\right|^{2}\left(1-|z|^{2}\right) d A(z) \\
& \quad \leq \sup _{I} \frac{\left(\log \frac{2}{\mid I}\right)^{2}}{|I|} \int_{S(I)} \frac{\left(1-|z|^{2}\right)\left|\varphi^{\prime}(z)\right|^{2}}{\left(1-|\varphi(z)|^{2}\right)^{2}\left(\log \frac{2}{1-|\varphi(z)|^{2}}\right)^{2}} d A(z)\|f\|_{B_{\log }}<+\infty
\end{aligned}
$$

Since $g \in B M O A_{\log }$ is equivalent to $\sup _{I} \frac{\left(\log \frac{2}{|I|}\right)^{2}}{|I|} \int_{S(I)}\left|g^{\prime}(z)\right|^{2}\left(1-|z|^{2}\right) d A(z)<+\infty$, we see $f \circ \varphi \in B M O A_{\log }$ for all $f \in B_{\log }$. 
Suppose that there exists a sequence $\left\{w_{n}\right\}_{n=1}^{\infty} \subset \partial D$ such that $\varphi(D) \subset \cup_{n=1}^{\infty}\{z \in D$ : $\left.\left|1-\overline{w_{n}} z\right|<\lambda\left(1-|z|^{2}\right)\right\}$ where $\lambda>0$ and that $\varphi(z)$ has $B_{\log }$-to-BMOA $A_{\log }$ pullback property. Put $f_{n}(z):=\log \left(\log \frac{2}{1-\bar{w} z}\right)$. Then it is clear that $f_{n} \in B_{\log }$. Let $G:=\cup_{n=1}^{\infty}\{z \in D:$ $\left.\left|1-\overline{w_{n}} z\right|<\lambda\left(1-|z|^{2}\right)\right\}$. Then for $z \in G$,

$$
\left(1-|z|^{2}\right)\left(\log \frac{2}{1-|z|^{2}}\right)\left|f_{n}^{\prime}(z)\right|=\frac{\left(1-\left|\overline{w_{n}} z\right|^{2}\right) \log \frac{2}{1-\left|\overline{\bar{w}_{n}} z\right|^{2}}}{\left|1-\overline{w_{n}} z\right|\left|\log \frac{2}{1-\overline{w_{n}} z}\right|} \geq C>0 .
$$

Since $\varphi(D) \subset \cup_{n=1}^{\infty}\left\{z \in D:\left|1-\overline{w_{n}} z\right|<\lambda\left(1-|z|^{2}\right)\right\}$, we have

$$
\left(1-|\varphi(z)|^{2}\right)\left(\log \frac{2}{1-|\varphi(z)|^{2}}\right)\left|f_{n}^{\prime}(\varphi(z))\right| \geq C>0 .
$$

Since $\varphi(z)$ has $B_{\log }$-to- $B M O A_{\log }$ pullback property and $f_{n} \in B_{\log }$, we have $f_{n} \circ \varphi \in B M O A_{\mathrm{log}}$. Hence we have

$$
\begin{aligned}
\sup _{I} \frac{\left(\log \frac{2}{|I|}\right)^{2}}{|I|} & \int_{S(I)} \frac{\left(1-|z|^{2}\right)\left|\varphi^{\prime}(z)\right|^{2}}{\left(1-|\varphi(z)|^{2}\right)^{2}\left(\log \frac{2}{1-|\varphi(z)|^{2}}\right)^{2}} d A(z) \\
& \leq \frac{1}{C} \sup _{I} \frac{\left(\log \frac{2}{|I|}\right)^{2}}{|I|} \int_{S(I)}\left|\left(f_{n} \circ \varphi\right)^{\prime}(z)\right|^{2}\left(1-|z|^{2}\right) d A(z)<\infty
\end{aligned}
$$

Acknowledgment. The author wishes to express his sincere gratitude to Professor Takahiko Nakazi for his many helpful suggestions and advices.

\section{References}

[1] Attele K.R.M., Toeplitz and Hankel on Bergman one space, Hokkaido Math. J. 21 (1992), 279-293.

[2] S.Axler, The Bergman space, the Bloch space, and comutators of multiplication operators, Duke Math.J.53(1986), 315-332.

[3] B.R.Choe, W.Ramey and D.Ullrich, Bloch-to-BMOA pullbacks on the disk, Pros. Amer. Math. Soc. 125 (1997), 2987-2996.

[4] Joseph Cima and David Stegenga, Hankel operators on $H^{p}$, Analysis at Urbana 1, London Mathematical Society, Lecture note series, Vol 137 ( Earl R. Berkson, N.T.Peck and J.Uhl, editors ), Camblidge[U.K.];New York: Camblidge Univercity Press, 1989, 133-150.

[5] C.C.Cowen and B.D.MacCluer, Composition Operators on Spaces of Analytic Functions, CRC Press, Boca Raton, 1995.

[6] J.Garnett, Bounded analytic functions, Academic Press, New York, 1981. 
[7] K.Madigan and A.Matheson, Compact composition operators on the Bloch space, Trans. Amer. Math.Soc. 347 (1995), 2679-2687.

[8] J.H.Shapiro, Composition Operators and Classical Function Theory, Springer-Verlag, New York, 1993.

[9] K.Zhu, Operator Theory in Function Spaces, Marcel Dekker, New York 1990.

[10] K.Zhu, Analytic Besov Spaces, J.Math.Anal.Appl.157(1991), 318-336.

Department of Mathematics

Hokkaido University

Sapporo 060-0810 ,Japan

yoneda @ math.sci.hokudai.ac.jp 